\title{
Investigation of a selectable multi-passband microwave photonic filter based on cascaded optical comb filters
}

\author{
F. WANG ${ }^{*}$, M.M. PENG ${ }^{2}$ \\ ${ }^{1}$ School of Physical Science and Technology, Southwest University, \\ Chongqing 400715, China \\ ${ }^{2}$ Institute of Science, Chongqing University of Technology, \\ Chongqing 400054, China
}

${ }^{*}$ Corresponding author: wangf17@swu.edu.cn

\begin{abstract}
A multi-passband microwave photonic filter (MPF) with selectable passband frequency, spanning $0-20 \mathrm{GHz}$ frequency range, is proposed, and experimentally demonstrated, in which passband frequency can be flexibly selected within a maximum passband number to four. The scheme is based on the generation of tunable optical comb lines using a broadband optical source sliced by cascaded optical comb filters, achieved by connecting an in-line birefringence fiber filter and a reconfigurable Lyot filter in series, such that various filter tap spacing and spectral combinations are obtained for the configuration of the MPF. The proposed MPF can operate with four different passband states, namely, single-, dual-, triple-, and quadruple-passband, only by adjusting polarization states of the cascaded optical comb filters. All these passbands are with a 3-dB bandwidth varying from 200 to $460 \mathrm{MHz}$ and more than 20-dB sidelobe suppression.
\end{abstract}

Keywords: microwave photonic filter, multi-passband filter, microwave signal processing.

\section{Introduction}

Microwave photonic filter (MPF) has a lot of practical applications in radio frequency (RF) signals processing [1 $\underline{-} \underline{3}]$. Compared with traditional microwave filter implemented in electrical domain, MPF has many unique features, such as low loss, large bandwidth, seamless tunability, good reconfigurability and immunity to electromagnetic interference (EMI) [4-7]. Nowadays, multiband communication is extremely essential in wireless and satellite communication systems [묘 $\underline{10}]$. A multi-passband MPF with flexibly reconfigurable passbands is urgently demanded for simultaneously selecting, processing, and transmitting the separated frequency channels. In recent years, multiband bandpass MPFs have aroused extensive research interest [11-19]. For example, a selectable multi -bandpass MPF based on a two-order high-birefringence fiber loop mirror (HB-FLM) was proposed, three selectable passbands with non-uniform bandwidth were achieved through the polarization state adjustment in the HB-FLM [11]. A MPF with tunable and switchable dual-passband by using a reflective fiber Mach-Zehnder interferom- 
eter (MZI) was presented, the switchable passband was achieved through rotating the polarization controllers (PCs) between the MZI and the fiber reflector, and the passband frequency could be tuned by changing the length of the variable optical delay line (VODL) in the MZI [12]. In addition, a high speed optically controlled MPF with dual-passband by using a Lyot loop as well as ultrafast nonlinear polarization rotation (NPR) effect in a semiconductor optical amplifier (SOA) was achieved, and by controlling the optical pump power, the MPF was switched between four different operation states [13].

In this paper, a multi-bandpass MPF whose passband frequency can be flexibly selected from one to four channels is proposed and experimentally demonstrated. The proposed MPF is based on a slicing broadband optical source (BOS) by using cascaded optical comb filters, in which an in-line birefringence fiber filter (IBFF) serves as the first-stage comb filter, and then a reconfigurable Lyot filter is used as the second-stage comb filter. The cascaded optical comb filters show various periodical spectral characteristics only utilizing two pieces of polarization maintaining fiber (PMF) with different length, which lead to the transmission spectrum of the filter with single or multiple periods. So, various filter tap spacing and spectral combinations are obtained for the configuration of the MPF. Then, the BOS slices are modulated by a phase modulator (PM) and time-delayed by dispersive medium. The frequency response of the MPF exhibits one or multiple passbands in accordance with the sampling periods. The experimental result shows that the proposed MPF is capable of operating with four different passband states, which are respectively the single-, dual-, triple-, and quadruple-passband, only by adjusting polarization states of the cascaded optical comb filters. All these passbands are with a 3-dB bandwidth varying from 200 to $460 \mathrm{MHz}$ and more than 20-dB sidelobe suppression. Furthermore, the frequency response on the baseband is suppressed.

\section{Experimental setup and principle}

The schematic diagram of the proposed multi-bandpass MPF is illustrated in Fig. 1. A broadband light emitted from a broadband optical source (BOS) via an optical iso-

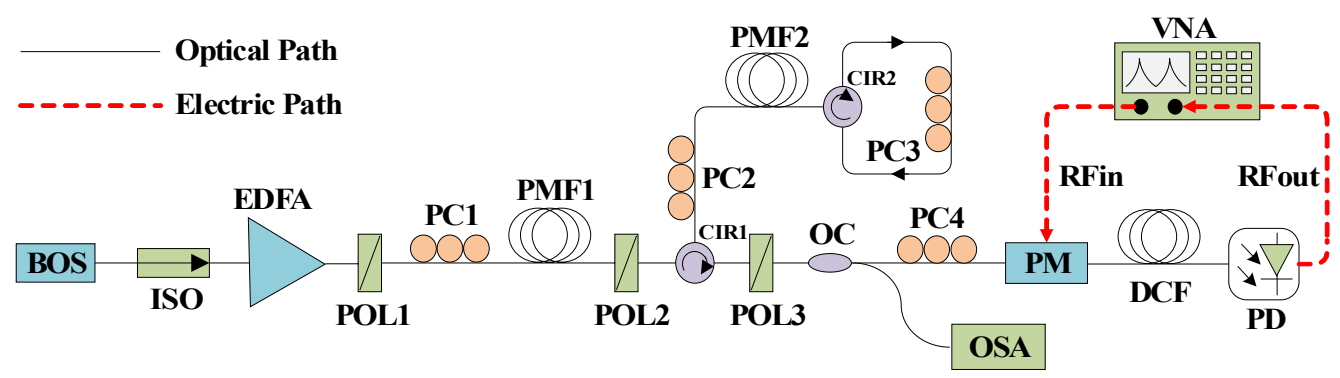

Fig. 1. The schematic diagram of the proposed multi-bandpass MPF with selectable passbands. BOS: broadband optical source, ISO: optical isolator, EDFA: erbium-doped fiber amplifier, POL: polarizer, PC: polarization controller, PMF: polarization maintaining fiber, CIR: circulator, OC: optical coupler, OSA: optical spectrum analyzer, PM: phase modulator, DCF: dispersion compensating fiber, PD: photodetector, and VNA: vector network analyzer. 
lator (ISO) is amplified by an erbium-doped fiber amplifier (EDFA), and firstly spectrum-sliced by the IBFF, which features less insertion loss than a normal HB-FLM [11] and better stability than an MZI [20] at room temperature. Then, a reconfigurable Lyot filter is connected to the output port of the IBFF through a circulator (CIR, CIR1) and acts as the second-stage comb filter to slice the optical spectrum again. The generated multi-wavelength comb lines are modulated by using a PM driven by a RF input signal. A coil of dispersion compensating fiber (DCF) is used to provide time delay for each wavelength (filter tap), and then the optical signal is fed into a photodetector (PD) and converted back to RF signal. A sweeping RF signal generated by a vector network analyzer (VNA) is used as the RF input for measuring the frequency response of the system.

Considering small signal modulation, only the first-order modulation sidebands are considered. The electrical field of each frequency component after the PM can be expressed as:

$$
E(\omega)=\exp (j \omega t)\left[1+m_{1} \exp (j \Omega t)+m_{2} \exp (-j \Omega t)\right]
$$

where $\Omega=2 \pi f$ is RF signal frequency. $m_{1}$ and $m_{2}$ are the modulation indexes for the two first-order modulation sidebands. Then the modulated signal travels through the dispersive medium with the transfer function of $H(\omega)=|H(\omega)| \exp (-j \varphi(\omega))$, the frequency response of the whole MPF can be derived as:

$$
H_{\mathrm{RF}}(\Omega)=\int T(\omega)\left[m_{1} H^{*}(\omega) H(\omega+\Omega)+m_{2} H(\omega) H^{*}(\omega-\Omega)\right] \mathrm{d} \omega
$$

$T(\omega)$ is the transmissivity function of the cascaded optical comb filters. Due to the dispersive medium acting as a phase shifter, we assume $H(\omega) \approx 1$. The phase dependence of the dispersive medium can be given by means of a Taylor expansion centered at $\omega_{0}$, as illustrated in Eq. (3),

$$
\varphi(\omega)=\varphi\left(\omega_{0}\right)+\tau_{\mathrm{g}}\left(\omega_{0}\right)\left(\omega-\omega_{0}\right)+\frac{1}{2} \beta L\left(\omega-\omega_{0}\right)^{2}+\frac{1}{3} \chi L\left(\omega-\omega_{0}\right)^{3}
$$

where $\tau_{\mathrm{g}}$ is the group delay, $\beta$ is the dispersion, $L$ is the length of dispersive medium and $\chi$ is the dispersion slope.

From the above equations, the frequency response of the MPF can be inferred as Eq. (4), from which one can see that there is a notch at baseband frequency.

$$
\begin{aligned}
& H_{\mathrm{RF}}(\Omega)=\exp \left[-j\left(\tau\left(\omega_{0}\right) \Omega+\frac{\chi L}{3} \Omega^{3}\right)\right] \\
& \times\left\{m_{1} \exp \left(-j \frac{B L}{2} \Omega^{2}\right) \int T(\omega) \exp \left[-j\left[\left(\beta \Omega+\chi \Omega^{2}\right)\left(\omega-\omega_{0}\right)+\chi\left(\omega-\omega_{0}\right)^{2} \Omega\right] L\right] \mathrm{d} \omega\right. \\
& \left.\quad+m_{2} \exp \left(+j \frac{B L}{2} \Omega^{2}\right) \int T(\omega) \exp \left[-j\left[\left(\beta \Omega-\chi \Omega^{2}\right)\left(\omega-\omega_{0}\right)+\chi\left(\omega-\omega_{0}\right)^{2} \Omega\right] L\right] \mathrm{d} \omega\right\}
\end{aligned}
$$


The passband center frequency $\Omega_{0}$ and the $3-\mathrm{dB}$ bandwidth $\Delta \Omega_{0}$ are determined by Eq. (5) and Eq. (6), respectively,

$$
\begin{aligned}
& \Omega_{0}=\frac{2 \pi}{\beta L \Delta \omega} \\
& \Delta \Omega_{0}=\frac{\sqrt{8 \ln 2}}{\beta L \delta_{\omega}}
\end{aligned}
$$

where $\Delta \omega$ is the optical comb spacing in angular frequency, which is related to the effective length $L$ of employed PMFs, $\delta_{\omega}$ represents the optical-source bandwidth [21] .

\section{Results and discussions}

The used BOS (COF-C/L-10-FC) is with a bandwidth of about $40 \mathrm{~nm}$ and a flat spectral distribution. The length of PMF1 and PMF2 are respectively 12 and $6 \mathrm{~m}$, with a birefringence of $4.0 \times 10^{-4}$. The PM (Photline MPZ-LN-20) has a 3-dB bandwidth of $20 \mathrm{GHz}$. The dispersion parameter and the length of DCF is $-140.57 \mathrm{ps} /(\mathrm{nm} \cdot \mathrm{km})$ and $2 \mathrm{~km}$, respectively. A high speeding PD ( $\mathrm{u}^{2} \mathrm{t}$ XPDV2120R) with a bandwidth of $50 \mathrm{GHz}$ is used to recover the microwave signals. The RF signal emitted from the VNA (CETC, AV3629D) is sweeping from $45 \mathrm{MHz}$ to $20 \mathrm{GHz}$, and the optical comb spectrum is monitored by an optical spectrum analyzer (OSA, Anritsu MS9740A).

\subsection{Single-passband MPF only using a IBFF}

The performance of the proposed MPF only using the first-stage optical comb filter (the IBFF) is firstly investigated. An IBFF is constructed by placing a PC and a piece of PMF between two polarizers (POLs). When the polarization orientation of the injected light is rotated $45^{\circ}$ with respect to fast axis (or slow axis) of the PMF (PMF1), phase difference between the two propagating fields on the fast and slow axes can be produced, then the two fields meet and interfere at POL2, so, a comb filter transmission function will be achieved from the IBFF. The comb spacing is determined by:

$$
\Delta \lambda=\frac{\lambda^{2}}{\Delta n l_{\text {eff }}}
$$

where $\Delta n$ and $l_{\text {eff }}$ is birefringence and the effective length of PMF, respectively. Using a 12-m long PMF in the IBFF, the spectrally sliced BOS with a single period is obtained, which is measured by the OSA with a resolution of $0.03 \mathrm{~nm}$, as shown in Fig. 2a. The inset is the corresponding optical comb spectrum with a 10-nm span for details, which is with a wavelength spacing of $0.5 \mathrm{~nm}$ and extinction ratio of over $10 \mathrm{~dB}$. Frequency response of the MPF is shown in Fig. 2b, measured by using the VNA. In 

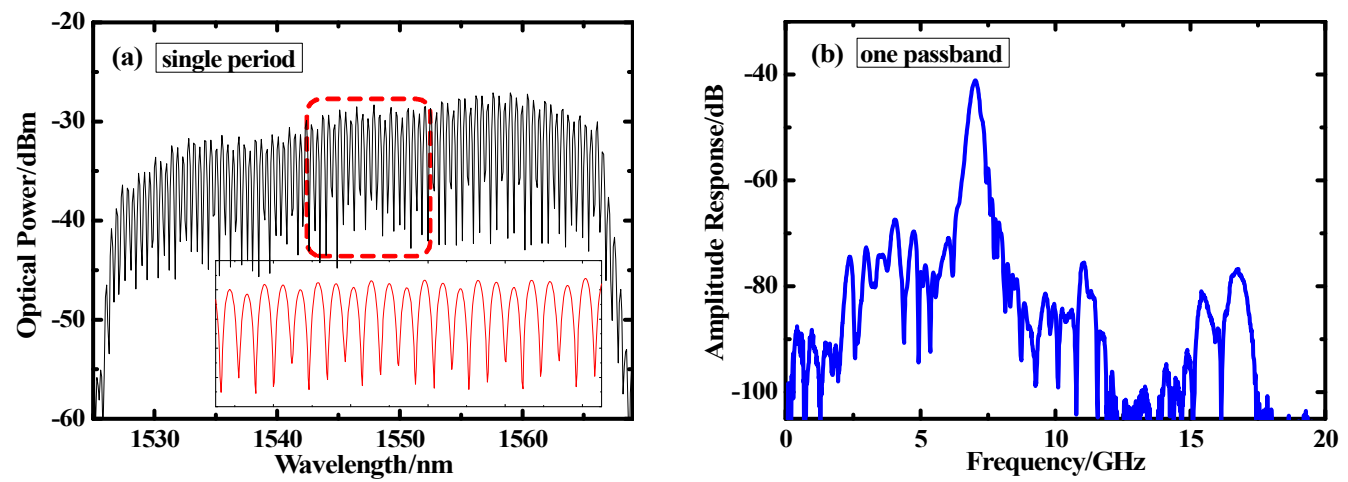

Fig. 2. (a) Experimentally measured optical spectrum with a single comb spacing of $0.5 \mathrm{~nm}$, the inset is the optical spectrum in $10 \mathrm{~nm}$ range for details, (b) the corresponding frequency response with a single passband at $7.06 \mathrm{GHz}$.

Fig. 2b, a single-passband frequency response is observed, and the passband center frequency is $7.06 \mathrm{GHz}$, with a $27-\mathrm{dB}$ suppression ratio.

\subsection{Switchable bandpass MPF only using a reconfigurable Lyot filter}

Then, the performance of the MPF only using a reconfigurable Lyot filter is investigated. As shown in Fig. 1, the reconfigurable Lyot filter mainly contains two POLs, two PCs, two CIRs and a piece of PMF (PMF2). The operation principle of the reconfigurable Lyot filter is illustrated in Fig. 3, which is designed by taking advantage of the bidirectional propagation in only one piece of PMF to adjust the effective length of PMF [22]. The polarization state of the input light after the POL2 is firstly adjusted by rotating PC 2 . Assume that the polarization direction of the input light is $45^{\circ}$ aligned with the fast axis of PMF2 with a physical length $L$. In the returning optical path, the polarization direction can be altered by rotating PC3, which results in the change of the effective length of PMF2. When the polarization direction of the incoming light after passing the $\mathrm{PC} 3$ is rotated either $45^{\circ}, 0^{\circ}$, or $-45^{\circ}$ with respect to the fast axis, the effective length

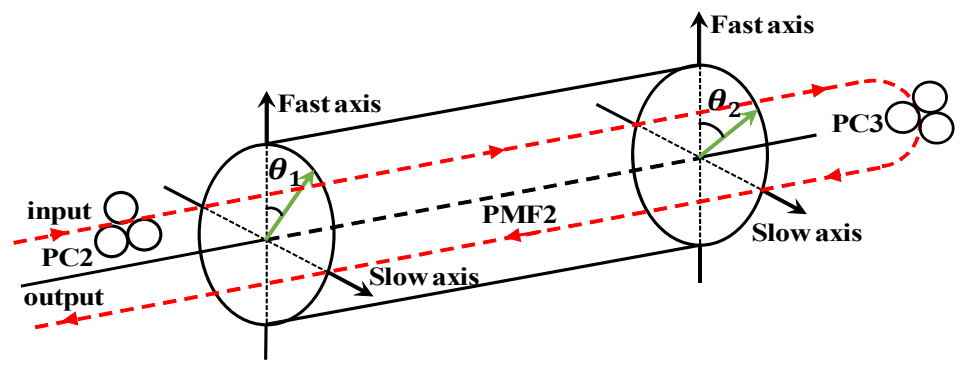

Fig. 3. The operation principle of the reconfigurable Lyot filter. 
of PMF2 is equal to $2 L, L$, or 0 , respectively. Hence, according to Eq. (7), switchable comb-spacing can be achieved.

Based on the above analysis, using a 6-m long PMF in the reconfigurable Lyot filter, optical combs with three different comb spacing combinations are obtained (interleaved 0.5 and $1 \mathrm{~nm}, 0.5 \mathrm{~nm}$, and $1 \mathrm{~nm}$ ) in Figs. $4 \mathbf{a}-4 \mathbf{c}$, corresponding to PMF 2 with an equivalent length of interleaved 12 and $6 \mathrm{~m}, 12 \mathrm{~m}$, and $6 \mathrm{~m}$, respectively. The extinction
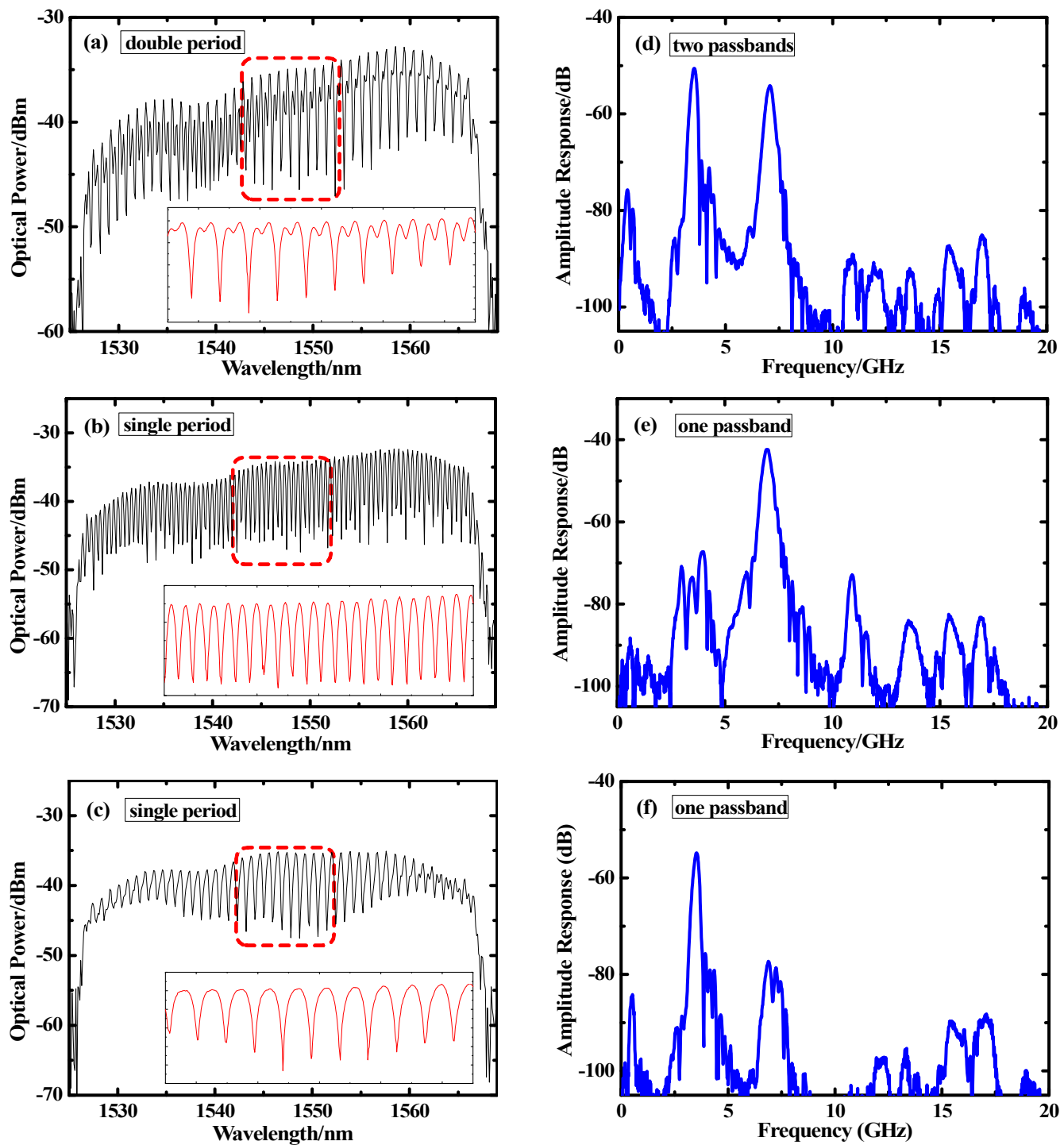

Fig. 4. Experimentally measured optical comb spectra, (a) with a double comb spacing of interleaved 0.5 and $1 \mathrm{~nm}$, (b) with a single comb spacing of $0.5 \mathrm{~nm}$, and (c) with a single comb spacing of $1 \mathrm{~nm}$; corresponding frequency responses of the multi-bandpass MPF in three different operating states: (d) dual passband at 3.53 and $7.06 \mathrm{GHz}$, (e) single passband at $7.06 \mathrm{GHz}$, and (f) single passband at $3.53 \mathrm{GHz}$. 
ratios of the optical combs are over $10 \mathrm{~dB}$ for all spectral combinations. Frequency responses of the MPFs corresponding to these optical combs are shown in Figs. $4 \mathbf{d}-4 \mathbf{f}$. In Fig. 4d, a dual passband at 3.53 and $7.06 \mathrm{GHz}$ is achieved. By simply adjusting the PCs in the reconfigurable Lyot filter, one of the two passbands can be suppressed, and a single passband at either 7.06 or $3.53 \mathrm{GHz}$ is achieved, respectively, as shown in Figs. $4 \mathbf{e}$ and $4 \mathbf{f}$. The suppression ratios are more than $20 \mathrm{~dB}$ for all the passbands in the experiment.

\subsection{Selectable multiband bandpass MPF using cascaded optical comb filters}

By cascading the reconfigurable Lyot filter with the IBFF, a reconfigurable high-order comb filter with multiple comb-spacing is achieved. The cascaded optical comb filters are analyzed, and the corresponding theoretical frequency is also deduced; the results are reported in the Table. One can see that there are seven typical combinations between the IBFF and the reconfigurable Lyot filter. Due to the limitations of experimental conditions, only a $12-\mathrm{m}$ and a $6-\mathrm{m}$ long of PMFs are available in the experiment, leading to the same effective lengths of the PMFs as well as optical comb spacing, for instance, combination 1 and combination 5, combination 2 and combination 4 . Thus, the passbands in these situations are overlapped. Furthermore, completely subtracting would make no passband generation, as combination 3 indicated. As a result, the simultaneous maximum passband number is limited to four.

T a b 1 e. The combinations of the cascaded optical comb filter $(\mathrm{PMF} 1=12 \mathrm{~m}, \mathrm{PMF} 2=6 \mathrm{~m})$.

\begin{tabular}{llllc}
\hline $\begin{array}{l}\text { Number } \\
1\end{array}$ & $\begin{array}{l}\text { Optical comb } \\
\text { combination }\end{array}$ & $\begin{array}{l}\text { Effective length } \\
\text { of PMF }[\mathrm{m}]\end{array}$ & $\begin{array}{l}\text { Comb spacing } \\
{[\mathrm{nm}]}\end{array}$ & $\begin{array}{l}\text { Theoretical passband } \\
\text { frequency [GHz] }\end{array}$ \\
\hline 2 & IBFF & 12 & 0.5 & 7.11 \\
3 & IBFF - Lyot1 & 6 & 1 & 3.55 \\
4 & IBFF - Lyot2 & 0 & None & None \\
5 & Lyot1 & 6 & 1 & 3.55 \\
6 & Lyot2 & 12 & 0.5 & 7.11 \\
7 & IBFF + Lyot1 & 18 & 0.33 & 10.66 \\
\hline
\end{tabular}

The measured optical comb spectra and corresponding frequency responses are shown in Fig. 5. Four different periodical spectral characteristics are clearly seen from Figs. 5a-5d, and the corresponding four different selectable passband states are also seen from Figs. $5 \mathbf{e}-\mathbf{5 f}$. Figure $5 \mathbf{e}$ gives a single-bandpass state which only contains one passband at $7.06 \mathrm{GHz}$, while two passbands at 7.06 and $14.12 \mathrm{GHz}$ are observed in Fig. 5f. Figure $5 \mathbf{g}$ shows a triple-passband state with three passbands at 3.53, 7.06 and $10.55 \mathrm{GHz}$ at the same time. A quadruple-passband state which simultaneously contains four passbands at 3.53, 7.06, 10.55 and $14.12 \mathrm{GHz}$ is observed in Fig. 5h. The experimental results show good agreements with the theoretical analysis. The $3-\mathrm{dB}$ bandwidth of all the passbands is within the range from 200 to $460 \mathrm{MHz}$, and all the passbands 
have more than $20 \mathrm{~dB}$ sidelobe suppression, providing good filtering characteristics. Since the passband selection depends on the polarization state adjustment in the cascaded optical comb filters, flexible and fast selectivity of the passband number can be conveniently achieved.

One can observe from Fig. 5 that, when using DCF to realize the phase-modulation to intensity-modulation conversion, carrier suppression effect (or dispersion-induced power fading effect) would degrade the amplitude uniformity of passbands at certain frequencies. Carrier suppression effect is caused by the double sideband modulation, which can be overcome by using single sideband modulation or other scheme, for example, using an all-optical microwave photonic filter combining a mode-locked fiber laser and a Mach-Zehnder structure in cascade to a $2 \times 1$ electro-optic modulator [23] . It is also worth noticing that the bandwidth of the passbands at lower frequency is narrower than that of the higher frequency. This is chiefly caused by a nonnegligible dispersion slop in the DCF [21], which could be mitigated by precisely designing the
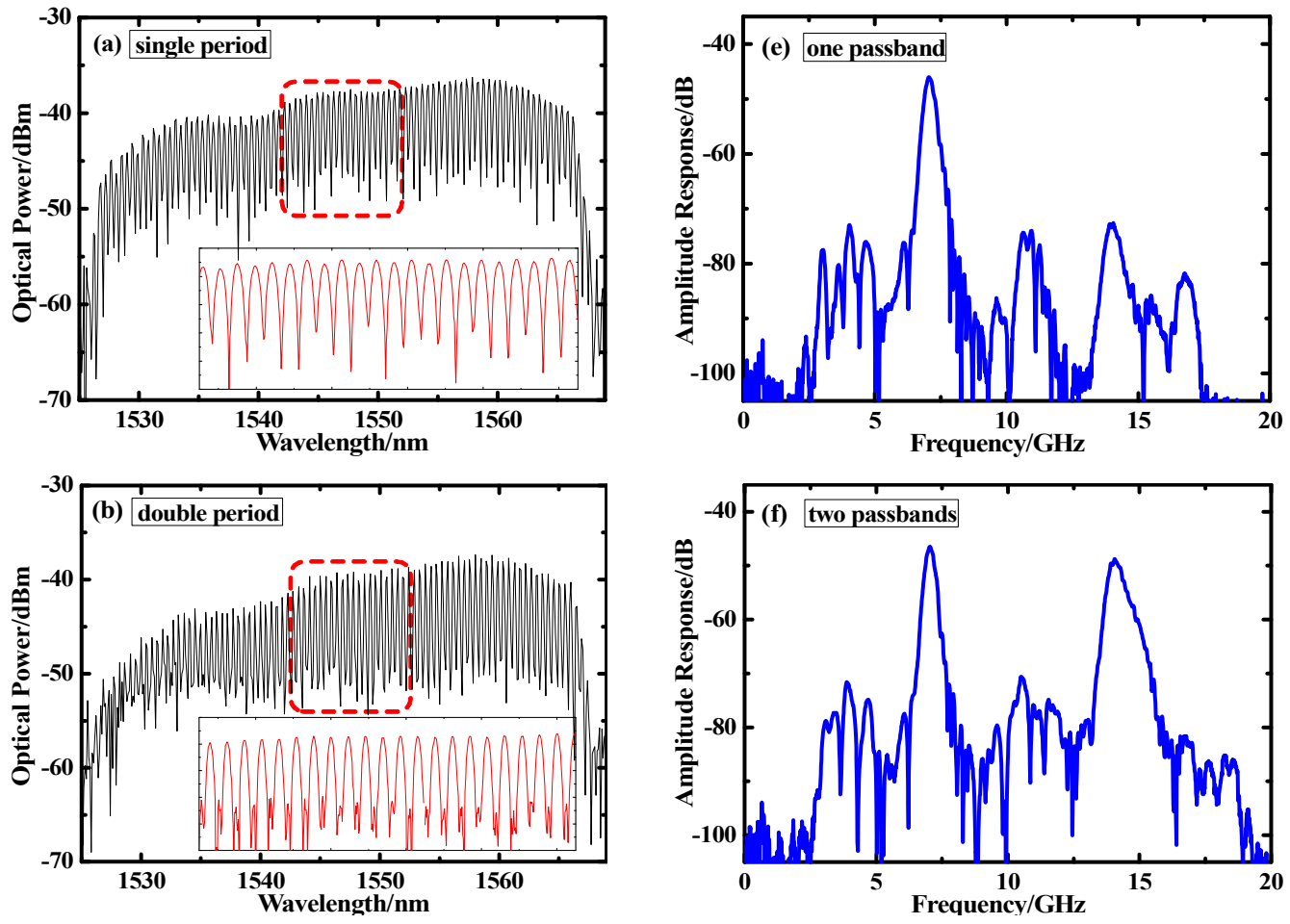

Fig. 5. Experimentally measured optical comb spectra, (a) with a single comb spacing of $0.5 \mathrm{~nm}$, (b) with a double comb spacing of interleaved 0.5 and $0.25 \mathrm{~nm}$, (c) with a triple comb spacing of interleaved $1,0.5$ and $0.33 \mathrm{~nm}$, and (d) with a quadruple comb spacing of interleaved $1,0.5,0.33$ and $0.25 \mathrm{~nm}$; corresponding frequency responses of the multi-bandpass MPF in four different operating states: (e) single passband at $7.06 \mathrm{GHz}$, (f) dual passband at 7.06 and $14.12 \mathrm{GHz}$, (g) triple passband at 3.53, 7.06 and $10.55 \mathrm{GHz}$, and (h) quadruple passband at 3.53, 7.06, 10.55 and $14.12 \mathrm{GHz}$. 

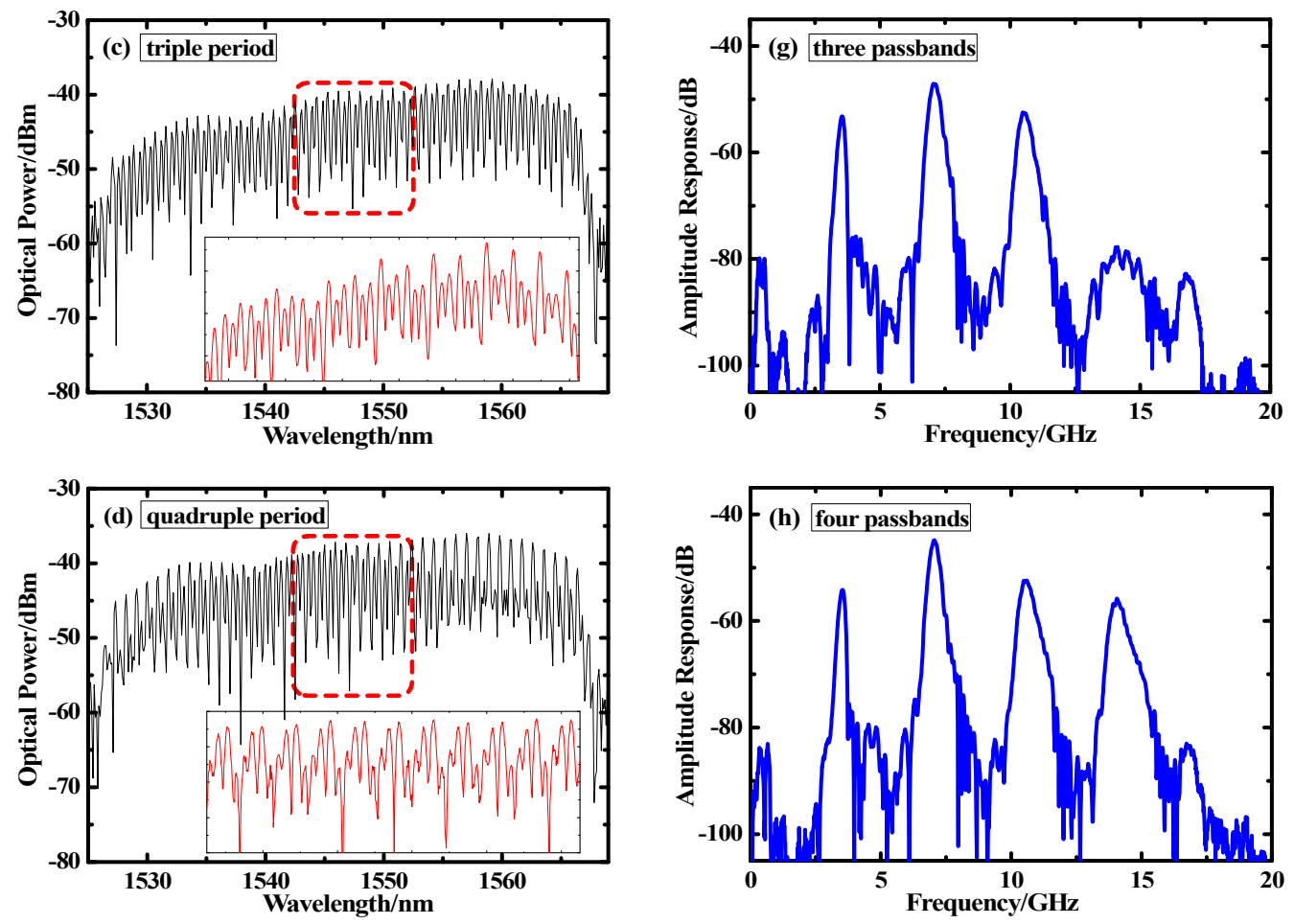

Fig. 5. Continued.

dispersion parameters of the dispersive medium in practical applications. In addition, a photonic crystal waveguide or a chirped fiber Bragg grating (CFBG) can be adopted to take the place of DCF $[\underline{23}, \underline{24}]$.

Furthermore, the maximum simultaneous passband number can be further increased by choosing more properly length of PMFs to avoid overlapping and cancelling of passbands, or cascading multistage optical comb filters in series to generate more interleaving optical comb $[25,26]$. Although the maximum simultaneous passband number and the reconfiguring operation can be further increased via multiple cascading combinations, size, complexity as well as loss of the whole system would be further increased, too. These problems can be properly solved by employing photonic integration techniques $[\underline{27}, \underline{28}]$.

\section{Conclusion}

A multi-bandpass MPF with selectable passbands is presented using a broadband optical source sliced by cascaded optical comb filters. Through polarization state adjustment, the proposed MPF can operate with four different passband states, which are the single-, dual-, triple-, and quadruple-passband state. The 3-dB bandwidth of all the 
passbands is within the range from 200 to $460 \mathrm{MHz}$, and all the passbands have more than $20 \mathrm{~dB}$ sidelobe suppression. The proposed multi-bandpass MPF exhibits the advantages of good tenability, stability, and flexibility, and is easy to implement, which has potential applications for $5 \mathrm{G}$ networks, radar systems, satellite systems and RF signal processing.

Acknowledgment - This work was supported by the Fundamental Research Funds for the Central Universities of China (SWU020001) and partly by the National Natural Science Foundation of China (61575034).

\section{References}

[1] YAo J., Photonics to the rescue: a fresh look at microwave photonic filters, IEEE Microwave Magazine 16(8), 2015, pp. 46-60, DOI: 10.1109/MMM.2015.2441594.

[2] Capmany J., Mora J., Gasulla I., Sancho J., Lloret J., Sales S., Microwave Photonic Signal Processing, Journal of Lightwave Technology 31(4), 2013, pp. 571-586, DOI: 10.1109/JLT.2012.2222348.

[3] Minasian R.A., Photonic signal processing of microwave signals, IEEE Transactions on Microwave Theory \& Techniques 54(2), 2006, pp. 832-846, DOI: 10.1109/TMTT.2005.863060.

[4] JiAng H., YAN L., MARPAUng D., Chip-based arbitrary radio-frequency photonic filter with algorithm-driven reconfigurable resolution, Optics Letters 43(3), 2018, pp. 415-418, DOI: 10.1364/ $\underline{\text { OL. } 43.000415 .}$.

[5] XU E., HAN X., Microwave photonic single-passband filter with highly flexible tunability of bandwidth and frequency, Optical Fiber Technology 33, 2017, pp. 51-55, DOI: 10.1016/j.yofte.2016.11.007.

[6] Stern Y., Zhong K., Schneider T., Zhang R., Ben-Ezra Y., Tur M., Zadok A., Tunable sharp and highly selective microwave-photonic band-pass filters based on stimulated Brillouin scattering, Photonics Research 2(4), 2014, pp. B18-B25, DOI: 10.1364/PRJ.2.000B18.

[7] Gao L., Zhang J., Chen X., Yaо J., Microwave photonic filter with two independently tunable passbands using a phase modulator and an equivalent phase-shifted fiber Bragg grating, IEEE Transactions on Microwave Theory and Techniques 62(2), 2014, pp. 380-387, DOI: 10.1109/TMTT.2013. 2294601.

[8] Borges R.M., Rodovalho T.N., Sodre A.C., Reconfigurable multi-band radio-frequency transceiver based on photonics technology for future optical wireless communications, IET Optoelectronics 9(5), 2015, pp. 257-262, DOI: 10.1049/iet-opt.2014.0128.

[9] Scotti F., Laghezza F., Ghelfi P., Valcarenghi L., Bogoni A., Wireless communications based on photonics-assisted multiband RF transceiver, 2014 OptoElectronics and Communication Conference and Australian Conference on Optical Fibre Technology, 2014, pp. 305-307.

[10] Laghezza F., Scotti F., Ghelfi P., Bogoni A., Photonics-assisted multiband RF transceiver for wireless communications, Journal of Lightwave Technology 32(16), 2014, pp. 2896-2904, DOI: 10.1109/ JLT.2014.2325959.

[11] Jiang Y., Shum P.P., Zu P., Zhou J., Bai G., Xu J., Zhou Z., Li H., Wang S., A selectable multiband bandpass microwave photonic filter, IEEE Photonics Journal 5(3), 2013, article 5500509, DOI: 10.1109/JPHOT.2013.2264663.

[12] Wu R., Chen H., Zhang S., Fu H., A switchable and tunable dual-passband microwave photonic filter, 2016 Progress in Electromagnetic Research Symposium (PIERS), 2016, pp. 1588-1591, DOI: 10.1109/PIERS.2016.7734720.

[13] GE J., FoK M.P., Optically controlled fast reconfigurable microwave photonic dual-band filter based on nonlinear polarization rotation, IEEE Transactions on Microwave Theory and Techniques 65(1), 2017, pp. 253-259, DOI: 10.1109/TMTT.2016.2614295.

[14] Wu R., Chen H., Zhang S., Fu H., Luo Z., Zhang L., Zhao M., Xu H., Cai Z., Tunable and selectable multipassband microwave photonic filter utilizing reflective and cascaded fiber Mach-Zehnder 
interferometers, Journal of Lightwave Technology 35(13), 2017, pp. 2660-2668, DOI: $10.1109 /$ JLT.2017.2665590.

[15] WeI X.H., Lu L.L., Research on microwave photonic filter with multiple passbands based on a broadband optical source, Optical Communication Technology 3, 2016, pp. 46-47, DOI: $10.13921 /$ j.cnki.issn1002-5561.2016.03.014.

[16] Xu Z., Fu H., Chen H., Xue H., Wu C., Huang C., Xu H., Cai Z., Zhang D., A tunable dual-passband microwave photonic filter based on optical slicing and dual-path fiber delay lines, Optics Communications 346, 2015, pp. 10-14, DOI: 10.1016/j.optcom.2015.01.073.

[17] Xu Z., Fu H., Chen H., Wu C., Xu H., Cai Z., Microwave photonic filter with two independently tunable passbands based on paralleled fiber Mach-Zehnder interferometers and dispersive medium, Applied Physics B 120, 2015, pp. 557-562, DOI: 10.1007/s00340-015-6165-2.

[18] Huang L., Chen D., Zhang F., Xiang P., Zhang T., Wang P., Lu L., Pu T., Chen X., Microwave photonic filter with multiple independently tunable passbands based on a broadband optical source, Optics Express 23(20), 2015, pp. 25539-25552, DOI: 10.1364/OE.23.025539.

[19] Chan E.H.W., Microwave photonic filter with a tunable nonperiodic multiple passband frequency response, Microwave and Optical Technology Letters 57(5), 2015, pp. 1089-1092, DOI: 10.1002/ mop.29024.

[20] Chen H., Xu Z., Fu H., Zhang S., Wu C., Wu H., Xu H., Cai Z., Switchable and tunable microwave frequency multiplication based on a dual-passband microwave photonic filter, Optics Express 23(8), 2015, pp. 9835-9843, DOI: 10.1364/OE.23.009835.

[21] Mora J., Ortega B., Diez A., Cruz J.L., Andres M.V., Capmany J., Pastor D., Photonic microwave tunable single-bandpass filter based on a Mach-Zehnder interferometer, Journal of Lightwave Technology 24(7), 2006, pp. 2500-2509, DOI: 10.1109/JLT.2006.874652.

[22] Luo A.P., Luo Z.C., Xu W.C., Channel-spacing switchable multi-wavelength fiber ring laser with one segment of polarization maintain fiber, Laser Physics Letters 6(8), 2009, pp. 598-601, DOI: 10.1002/lapl.200910041.

[23] Mora J., Ortigosa-Blanch A., Pastor D., Capmany J., Tunable microwave photonic filter free from baseband and carrier suppression effect not requiring single sideband modulation using a Mach -Zehnder configuration, Optics Express 14(17), 2006, pp. 7960-7965, DOI: 10.1364/OE.14.007960.

[24] Sancho J., Bourderionnet J., Lloret J., Combrié S., Gasulla I., Xavier S., Sales S., Colman P., Lehoucq G., Dolfi D., Capmany J., De Rossi A., Integrable microwave filter based on a photonic crystal delay line, Nature Communications 3, 2012, article 1075, DOI: 10.1038/ncomms2092.

[25] Shahoеi H., Li M., Yao J., Continuously tunable time delay using an optically pumped linear chirped fiber Bragg grating, Journal of Lightwave Technology 29(10), 2011, pp. 1465-1472, DOI: 10.1109 / JLT.2011.2132754.

[26] GE J., FoK M.P., Continuously tunable and reconfigurable microwave photonic multiband filter based on cascaded MZIs, 2017 IEEE Photonics Conference (IPC), pp. 381-382, DOI: 10.1109/IPCon. 2017.8116149.

[27] FоK M.P., GE J., Tunable multiband microwave photonic filters, Photonics 4(4), 2017, article 45, DOI: $10.3390 /$ photonics4040045.

[28] Pérez D., Gasulla I., Crudgington L., Thomson D.J., Khokhar A.Z., Li K., Cao W., Mashanovich G.Z., CAPMANY J., Multipurpose silicon photonics signal processor core, Nature Communications 8, 2017, article 636, DOI: 10.1038/s41467-017-00714-1.

[29] Zhuang L., Roeloffzen C.G.H., Hoekman M., Boller K.-J., Lowery A.J., Programmable photonic signal processor chip for radiofrequency applications, Optica 2(10), 2015, pp. 854-859, DOI: 10.1364/OPTICA.2.000854. 